\title{
Development of a Conceptual View of a Patron Queuing System in TCP / IPs
}

\author{
Dr. T.C.Manjunath \\ Ph.D. ( IIT Bombay ), Member IEEE \& Fellow IETE \\ PRINCIPAL, Atria Institute of Technology, \\ Bangalore, Karnataka, India
}

\author{
Reema Sharma \\ Sr. Lecturer, \\ Dept. of ECE, Oxford College of Engg, \\ Bangalore-87
}

\begin{abstract}
This paper presents a brief overview of a patron queuing system in the communication sectors. Businesses have been faced with an ever-increasing demand from customers for convenient services. Customers do not want to spend their time waiting in a long queue. They want quick service because time is money \& money is business. In order to meet this demand, service-based businesses need logistic data to help their operations run smoothly. The Patron Queuing System (PQS) counts the number of people waiting in the queue. It can then relay this number to the potential customer via website, PDA (Personal Digital Assistant). The PQS is installed in a location that ensures accurate data collection. More specifically, the sensor detects one person entering the queue at a time. The number of people entering and exiting the queue will be measured. The number of people in the queue will be relayed to the potential customer. There is a time delay when sending data from the PQS to the potential customer for viewing. The accuracy of the PQS is measured by comparing the data corresponding to the number of people in the queue at a certain time to the actual, real-time number of people in the queue. The time delay is not too long, as it would affect the accuracy and reliability of the PQS. The PQS has two user interfaces. One is for the potential customer, which is easy to read and accessible to all. The other is at the business and provides them with a way to know that the PQS is functioning properly. Internet connection from the business is necessary for the data to be sent from the PQS to the potential customer. The PQS operates in a wide range of temperatures and humidity. It is unobtrusive and unnoticeable to the patron and attempts to blend the PQS with the environment to reduce the risk of tampering. This review paper makes a brief survey of the queuing system concepts and the readers can make this as a base paper and do some extensive research in this exciting field.
\end{abstract}

\section{General Terms \\ PQS, PDA}

\section{Keywords}

Virtual keyboards, Wireless technologies

\section{INTRODUCTION}

In today and age, technology has found its way into almost all facets of life. It has improved our quality of life by making once arduous and time consuming tasks extremely easy and convenient. Technology has helped people manage their time and lives better. This is a driving force behind the Patron Queuing System (PQS). Since the Industrial Revolution, businesses have been faced with an ever-increasing demand from customers for convenient services. Customers do not want to spend their time waiting in a long line. They want quick service because time is money. In order to meet this demand, service-based businesses need logistic data to help their operations run smoothly.

The Senior Design Project 2004 (SDP '04) team advised by Professor Gong [1], has created a solution to this problem by designing the Patron Queuing System (PQS). This device counts the number of people waiting in a queue. It can then relay this number to the potential customer. The PQS created by SDP ' 04 team has never been applied in the business environment. We plan on implementing the PQS at several businesses, evaluating its performance, and enhancing this device with feedback from the businesses and patrons [1].

The Patron Queuing System will appeal to most businesses. It is intended to provide them with data trends that can help prepare for various customer loads, which in turn will help them predict how much staffing is required. Businesses will save money if they don't have to deal with employer conflicts of schedules by using these data to help them plan ahead. The PQS system should be installed in an environment where not more than one person is passing the sensors at a time. This will enforce accurate data and will directly affect its reliability. There is very little maintenance required of the owner of the PQS. This document is the requirement specification for an electronic device to aid in the implementation and proper use of the PQS.

It will use technology to provide users with up to the minute information about businesses and other establishment's current occupancy as well as other useful information. People will be able to access this information from a few ways. They can either go to a website or use their cell phone to text message to the server but that will relay the information requested. The Patron Queuing System appeals most businesses. It is intended to provide them with data trends that can help prepare for various customer loads, which in turn helps them predict how much staffing is required [2].

Businesses will save money if they don't have to deal with employer conflicts of schedules by using these data to help them plan ahead. At the establishments, a sensor will be installed that will keep track of the amount of people currently at the location. 
The data that the sensor records will then be sent to the server via the internet and then be added to the database. Based on the data the patron receives, it can make time saving choices about their next course of action.

The PQS is installed in an environment where not more than one person is passing the sensors at a time. This will enforce accurate data and will directly affect its reliability. There is very little maintenance required for the owner of the PQS [3]. The PQS works as follows: A user will want to know the current occupancy of a specific location. They can use their cell phones to text message a bot on the server. A menu will show up letting them see their choices. They can then send a number reply back to the bot. The bot then accesses the database storing PQS data and retrieves the information to the user. The data will be placed into the database via a client-side program. The program will record the number of the times the sensor is triggered, indicating current occupancy. This data is sent and stored on the server's database is what will be displayed on the cell phone or the web.

The paper is organized in the following sequence. A brief review about the queuing systems was presented in the previous paragraphs. Section 2 presents an overview about the personal digital assistance, i.e., PDA's. Section 3 deals with the features of the PQS. The protocols are discussed in section 4 . Further, the concept of layers is presented in section 5. RS232 used in PQS is presented in the next section. MYSQUL in section 7 is presented next. The operation of the PQS is presented in section 8. This is followed by the conclusions in the section 9 and the references.

\section{Personal Digital Assistance (PDA)}

A Personal Digital Assistant (PDA) is a handheld computer, also known as a palmtop computer. Newer PDA's also have both color screens and audio capabilities, enabling then to be used as mobile phones, web browsers, or portable media players. Many PDA's can access the Internet, intranets or extranets via Wi-Fi, or Wireless Wide Area Networks (WWANs). Many PDA's employ touch screen technology.

The first PDA is considered to be the CASIO PF-3000 released in May 1983. The term was first used on January 7, 1992 by Apple Computer CEO John Sculley. In 1996 Nokia introduced the first mobile phone with full PDA functionality, the 9000 Communicator, which has since grown to become the world's best selling PDA and which spawned a category of phones called the smartphone.Today the vast majority of all PDAs are smartphones, selling over 150 million units while non-phone ("stand-alone") PDAs sell only about 3 million units per year. The RIM Blackberry, the Apple iPhone and the Nokia N-Series are typical smart-phones.

\section{Features}

A typical PDA has a touch screen for entering data, a memory card slot for data storage and at least one of the following for connectivity Bluetooth and/or WiFi. However, many PDA's (typically those used primarily as telephones) may not have a touch screen, using soft-keys, a directional pad and either the numeric keypad or a thumb keyboard for input. Software typically required to be a PDA includes an appointment calendar, a to-do list, an address book for contacts and some sort of note program [4].

Connected PDA's also typically include E-mail and Web support.Touch screen: Many original PDAs, such as the Apple Newton and Palm Pilot, featured touch screens for user interaction, having only a few buttons usually reserved for shortcuts to often used programs. Touch screen PDAs, including Windows Pocket PC devices, usually have a detachable stylus that can be used on the touch screen. Interaction is then done by tapping the screen to activate buttons or menu choices, and dragging the stylus to, for example, highlight. Text input is usually done in one of four ways [5]:

1. Using a virtual keyboard, where a keyboard is shown on the touch screen. Input is done by tapping letters on the screen.

2. Using external keyboard or corded keyboard connected by USB, IR or Bluetooth.

3. Using letter or word recognition, where letters or words are written on the touch screen, and then "translated" to letters in the currently activated text field.

4. Stroke recognition, in this system a predefined set of strokes represents the various characters needed. The user learns to draw these strokes on the screen or in an input area. The strokes are often simplified character shapes to make them easier to remember.

Memory cards: Although many early PDAs did not have memory card slots now most have either an SD (Secure Digital) and/or a Compact Flash slot. Although originally designed for memory and Compact Flash cards are available for such things as Wi-Fi and Webcams. Some PDAs are now compatible with micro SD cards, which are physically much smaller than standard SD cards.

Wireless connectivity: Most modern [6] PDAs have Bluetooth wireless connectivity, an increasingly popular tool for mobile devices. It can be used to connect keyboards, headsets, GPS and many other accessories, as well as sending files between PDAs. Many mid-range and superior PDAs have Wi-Fi/WLAN/802.11connectivity, used for connecting to Wi-Fi hotspots or wireless networks.

Older PDAs predominantly have an IrDA (infrared) port; however fewer current models have the technology, as it is slowly being phased out due to support for Bluetooth and Wi-Fi. IrDA allows communication between two PDAs: a PDA and any device with an IrDA port or adapter. Most universal PDA keyboards use infrared technology because many older PDAs have it, and infrared technology is low-cost and has the advantage of being permitted aboard aircraft.

Synchronization: An important function of PDAs is synchronizing data with a PC. This allows up-to-date contact information stored on software such as Microsoft Outlook to update the database on the PDA. The data synchronization ensures that the PDA has an accurate list of contacts, appointments and e-mail, allowing users to access the same information on the PDA as the host computer. The synchronizing also prevents the loss of information stored on the device in case it is lost, stolen, or destroyed. Another advantage is that data input is usually a lot quicker on a PC, 
since text input via a touch screen is still not quite optimal. Transferring data to a PDA via the computer is therefore a lot quicker than having to manually input all data on the handheld device.

Uses Automobile navigation: Many PDAs are used in car kits and are fitted with differential Global Positioning System (GPS) receivers to provide real-time automobile navigation.

Medical and scientific uses: PDAs have been shown to aid diagnosis and drug selection and some studies have concluded that their use by patients to record symptoms improves the effectiveness of communication with hospitals during follow-up.

Educational uses: Personal computing has become a vital learning tool. With the capabilities of PDAs, teachers are now able to provide a collaborative learning experience for their students.

Sporting uses: PDAs are used by glider pilots for pre-flight planning and to assist navigation in cross-country competitions. They are linked to a GPS to produce moving-map displays showing the tracks to turn-points, airspace hazards and other tactical information. PDAs may also be used by music enthusiasts. The EO was an early commercial tablet computer created by AT\&T .It was more similar to a large personal digital assistant with wireless communications.

\section{Protocols used in PQS}

The Internet Protocol suite (commonly known as TCP/IP) is the set of communications protocols used for the Internet and other similar networks. It is named from two of the most important protocols in it: the Transmission Control Protocol (TCP) and the Internet Protocol (IP), which were the first two networking protocols defined in this standard.

The Internet Protocol Suite, is a set of layers. Each layer solves a set of problems involving the transmission of data, and provides a well-defined service to the upper layer protocols based on using services from some lower layers. Upper layers are logically closer to the user and deal with more abstract data, relying on lower layer protocols to translate data into forms that can eventually be physically transmitted. The TCP/IP model consists of four layers. From lowest to highest, these are the Link Layer, the Internet Layer, the Transport Layer, and the Application Layer [6].

\section{The concept of layers}

The TCP/IP uses networking to provide abstraction of protocols and services. In general, an application (the highest level of the model) uses a set of protocols to send its data down the layers, being further networked at each level.The TCP is a transportlevel protocol that sits on top of the IP. It provides a reliable fullduplex byte stream between processes, whether they reside on the same machine or different machines [7].

TCP is responsible for ensuring that the packets transmitted are received in the same order in which they were sent out, and reconstructed into the original byte stream. This is important, since two adjacent packets could take different routes to the same destination. The functional groups of protocols and methods are the Application Layer, the Transport Layer, the Internet Layer, and the Link Layer. Internet Protocol is a public standard network layer protocol. It is responsible for getting data from one machine to another, but is not responsible for the assembling of that data for the receiving process [8].

MAC address: Media Access Control address (MAC address), Ethernet Hardware Address (EHA), hardware address, adapter address or physical address is a quasi-unique identifier assigned to most network adapters or network interface cards (NICs) by the manufacturer for identification. If assigned by the manufacturer, a MAC address usually encodes the manufacturer's registered identification number. A network card, network adapter, network interface controller (NIC), network interface card, or LAN adapter is a computer hardware component designed to allow computers to communicate over a computer network [9].

It provides physical access to a networking medium and provides a low-level addressing system through the use of MAC addresses. It allows users to connect to each other either by using cables or wirelessly. In TCP/IP networks, the MAC address of a subnet interface can be queried with the IP address using the Address Resolution Protocol (ARP) for Internet Protocol. On broadcast networks, such as Ethernet, the MAC address uniquely identifies each node and allows frames to be marked for specific hosts. It thus forms the basis of most of the Link layer networking upon which upper layer protocols rely to produce complex, functioning networks.

\section{RS232 used in Pattern Queuing Systems}

RS232 (Recommended Standard 232) is a standard for serial binary data signals connecting between a DTE (Data Terminal Equipment) and a DCE (Data Circuit-terminating Equipment). It is commonly used in computer serial ports. RS-232-compatible port is a standard feature for serial communications, such as modem connections, on many computers. In RS-232, user data is sent as a time-series of bits. Both synchronous and asynchronous transmissions are supported by the standard [10].

In addition to the data circuits, the standard defines a number of control circuits used to manage the connection between the DTE and DCE. Each data or control circuit only operates in one direction, that is, signaling from a DTE to the attached DCE or the reverse. Since transmit data and receive data are separate circuits, the interface can operate in a full duplex manner, supporting concurrent data flow in both directions. The standard does not define character framing within the data stream, or character encoding.

\section{MYSQL used in PQS}

The MYSQL is a relational database management system which has become the world's most popular open source database because of its consistent fast performance, high reliability and ease of use. The program runs as a server providing multi-user access to a number of databases. MYSQL runs on more than twenty platforms including Linux, Windows, OS/X, giving the kind of flexibility that gives total control. There is only one MYSQL server package, which includes a mysqld binary with all functionality and storage engines enabled. Instead of providing a separate debug package, a server binary with extended debugging information is also included in the standard package. There are 3 types of MYSQL server compilations. 


\section{Operation of the PQS}

The principle of operation of the PQS is explained as follows : The PQS is mounted at one end of the queue enabling the collection of data of people coming in and out of the queue. Two sensors are used to detect when a customer enters or leaves the line of a business and the microcontroller will process this data to calculate the number of people in the queue. This data is sent to a database for storage and display for the potential customer. The information provided by the PQS is made available by a website, PDA, AIM robot buddy, automated phone number, or it is also set up at the business for display.

1. User interface: A website or AIM robot buddy is used by the potential customer to view the number of people waiting in the queue at a particular business.

2. Input: The input to the PQS consists of an interruption of the infrared signal to the sensors from patrons entering and leaving a queue.

3. Output: The output of PQS is a number displayed on an internet website indicating the number of people in line.

The setting up of the PQS is further explained as follows :

1. The PQS is setup at the entrance of the business or a place where there is a lot of customer activity.

2. The PQS is to be hooked to a power socket.

3. Verify that wireless internet exists at the business site.

4. Power up the computer and check to see if the computer can detect the Serial LAN. The Serial LAN is assigned an IP address similar to that of the computer.

Once the setup is finished, the data is collected from the computer.

The PQS is camouflaged with the atmosphere to reduce the risk of patron intervention, which may lead to damage. To implement whether someone was entering or exiting a business, two sensors are setup in parallel. If one sensor is triggered before the other, then it is known that a customer is passing the sensor in a particular direction.

The mid-range PIC microcontroller is used to add and store the count it receives from the sensors. The PIC enables us to interface with any device via a serial port. The total count (patrons entering line minus exiting line) of the queue is sent to the serial port device every 30 seconds.

The Serial LAN is used to connect the PIC to the Internet wirelessly. This device converts the RS232 packets to the TCP/IP packets and sends them out at $11 \mathrm{Mbps}$. The device has a static IP and MAC address. It is fully functional wirelessly and has options for customization.

\section{Conclusions}

This review paper about the PQS presented a brief overview of the public queuing systems \& its basic concepts in the planning, operation, design of the same.

\section{Acknowledgements}

The authors are very much thankful to the staff $\&$ management of Oxford college of engg. For their cooperation \& help rendered regarding this work.

\section{REFERENCES}

[1] Mark DiBenedetto, Aziz El-Tatar, Kalyan Sunkara, Syed Ahmed Khan, Weibo Gong, "Patron Queuing System, Requirement Specifications”, SDP 05, October 17, 2004.

[2] Pamela R.Lessing, First Week with My New Digital Organiser, A Very Basic Guide to Palm OS PDAs, Capital Books, 2004.

[3] Daryl Wilding McBride, Java on PDAs with J2ME, Addison Wesley, 2003.

[4] Andrew Tanenbaum, Computer Networks, 4th Edn, 2002.

[5] Andrew Tanenbaum, Albert S Woodhall, Operating System Design and Implementation, 3rd Edn, Prentice Hall, 2006.

[6] Larry L Peterson, Bruce S. Davie, Computer Networks: A Systems Approach, Fourth Edition (The Morgan Kaufmann Series in Networking), 4th Edn, 2007.

[7] Wai K.Ching, Iterative Methods for Queuing and Manufacturing Systems, Springer, 2001.

[8] Seyed Tahaghoghi, Hugh Willaims, Learning MySQL, Illustrated Edn, 2006.

[9] Paul DuBois, MySQL Cookbook, O'Reilly Media, Inc.; 2 edition,2006.

[10] David Pogue, MAC OS X Leopard: The Missing Manual,Pogue Press, 2007.

Mrs. Reema Sharma was born in Batala, Dist. Gurdaspur, Punjab, India on Apr. 4, 1980 \& received the B.Tech. Degree from the prestigious Punjab Technical University (PTU) in Jalandhar, Punjab in the year 2001 in First Class and M.Tech. in Electronics \& Communication Engg. from the prestigious National Institute of Technology Kurukshetra (formerly, Regional Engg. College) in 2005 in First Class with distinction, respectively. She has got an industrial experience of 1 year and teaching experience of more than 5 years in various engineering colleges all over the country and is currently working as a Lecturer in the Department of Electronics and Communication Engineering in Oxford College of Engineering in Bangalore, Karnataka, India, which is affiliated to Visvesvaraya Technological University, Belgaum. She has also participated, coordinated in a number of workshops \& other events. She has also guided few projects $\&$ presented few papers in national \& international events.

Dr. T.C.Manjunath was born in Bangalore, Karnataka, India on Feb. 6, 1967 \& received the B.E. Degree (Bachelor of Engg.) from R.V. College of Engg. (Bangalore University) with M.Tech. degree from L.D. College of Engg. (Gujarat University) and Ph.D. from the prestigious Indian Institute of Technology Bombay (IIT Bombay), respectively. He has got a teaching experience of nearly 21 long years in various engineering colleges all over the country (Karnataka, Gujarat, Maharashtra) and is currently working as PRINCIPAL in ATRIA Institute of Technology, Bangalore, Karnataka, India. He has also worked as a Project Assistant and as a Research Engineer in the Systems and Control Engineering (IIT Bombay, India) and worked on control of space launch vehicles using FOS feedback technique. $\mathrm{He}$ has published a number of papers in various National, International journals and Conferences and published three textbooks on Robotics. 